\title{
An Explicit Nodal Space-Time Discontinuous Galerkin Method for Maxwell's Equations
}

\author{
L. D. Angulo, J. Alvarez, Member, IEEE, M. F. Pantoja, Senior Member, IEEE, and S. G. Garcia, Member, IEEE
}

\begin{abstract}
A novel implicit nodal Space-Time Discontinuous Galerkin (STDG) method is proposed in this paper. An eigenvalue analysis is performed and compared with that for a DG scheme solved with a 4th-Order Runge-Kutta time integrator. We show that STDG offers a significant improvement of dissipative and dispersive properties and allows larger time steps, regardless of the spatial hp-refinement. A domain-decomposition technique is used to introduce an explicit formulation of the method in order to render it computationally efficient.
\end{abstract}

Index Terms-Discontinuous Galerkin Methods, Computational Electrodynamics, Space-Time Discontinuous Galerkin

\section{INTRODUCTION}

A common approach for Discontinuous Galerkin (DG) timedomain (TD) methods, is to treat the time and space variables separately [1], often using an explicit time-integrating schemes such as the 4th-Order Low-Storage Runge-Kutta LSERK4, which prevents the full exploitation of the higher-order spatial convergence. Although works to cope with this limitation [2] exist, a noteworthy alternative is to use Space-Time DG (STDG) methods, already used in other fields of Physics [3], [4], and in Electromagnetics [5], [6]. A major drawback of STDG resides in its implicit nature, though semi-explicit approaches also exist [5], [6] (tent-pitching technique).

In this work, we present a novel STDG formulation combined with a causal domain-decomposition technique [7] to render it explicit (E-STDG). This letter is organized as follows: we first formulate a nodal [1] STDG scheme, with a new spurious-free upwind-in-space flux, combined with a centeredin-time flux. Next, we study the properties of the resulting implicit STDG scheme with an eigenvalue analysis, comparing with a DG-LSERK4 one. Next, we describe the explicit causal formulation E-STDG, and validate it with a simple resonant problem. We finally conclude that the use a nodal approach, together with the domain-decomposition technique provides an affordable solution to the problem.

\section{IMPLICIT FORMULATION}

Let us start by defining a 2D space-time region tessellated with $k=1, \ldots, K_{x}$ elements. With $\zeta \in V$ defined as a spacetime coordinate within each element, and with $\hat{n}=\left(n_{t}, n_{x}\right)$

This work is partially funded by the National Projects TEC2010-20841C04-04, TEC2013-48414-C3-01, CSD2008-00068, P09-TIC-5327, P12-TIC1442 , and from the GENIL excellence network.

The $1^{\text {st }}, 3^{\text {rd }}, 4^{\text {th }}$ authors are with the Dept. of Electromagnetism, Univ Granada, 18071 Granada, Spain, (Corresponding: salva@ugr.es). The $2^{\text {nd }}$ is with Airbus Defense and Space, 28906 Getafe, Spain.

The authors thanks Stephane Lanteri, Sascha Schnepp and Martin Lilienthal for their comments, discussions and support. normal vectors pointing outwards from its boundary. The weak form of 1D Maxwell's curl equations is found by multiplying by weighting functions $\alpha_{i j}(\zeta)$, integrating over $V$, and enforcing the residual to vanish. For instance, the freespace Ampère's law (taking for simplicity the permittivity and permeability both equal to 1) becomes

$$
\int_{V}\left(\partial_{t} E(\zeta)+\partial_{x} H(\zeta)\right) \alpha_{i j}(\zeta) d \zeta=0
$$

with $E$ and $H$ being the electric and magnetic fields. Integrating by parts in (1) and replacing the boundary flux-integral by a numerical flux, as usual in DGTD, we find

$$
\int_{V} \partial_{x} H \alpha_{i j} d \zeta=\int_{T} d t\left[\int_{\partial X} \hat{n} \cdot H^{*} \alpha_{i j} d x-\int_{X} H \partial_{x} \alpha_{i j} d x\right]
$$

with $H^{*}$ (and similarly $E^{*}$ ) being the usual numerical fluxes [1]. Next, following the Galerkin procedure, we expand the fields in series using the weighting functions also as basis functions, and assuming that their space-time dependence can be separated in polynomials of orders $P^{t}$ and $P^{x}$ for the temporal and spatial parts, respectively

$$
H^{h}(\zeta)=\sum_{i=0}^{P^{t}} \sum_{j=0}^{P^{x}} \alpha_{i j}(\zeta) H_{i j}=\sum_{i=0}^{P^{t}} \sum_{j=0}^{P^{x}} \alpha_{i}^{t}(t) \alpha_{j}^{x}(x) H_{i j}
$$

and similarly for E. Substituting (3) into (2), the spatialstiffness term becomes,

$$
\int_{V} H^{h} \partial_{x} \alpha_{m n} d \zeta=\sum_{i=0}^{P^{t}} \sum_{j=0}^{P^{x}} H_{i j} \int_{T} \alpha_{i}^{t} \alpha_{m}^{t} d t \int_{X} \alpha_{j}^{x} \partial_{x} \alpha_{n}^{x} d x
$$

And the spatial-flux term,

$$
\begin{gathered}
\int_{T}\left(H^{*}\left(t, x_{R}\right) \alpha_{m n}\left(t, x_{R}\right)-H^{*}\left(t, x_{L}\right) \alpha_{m n}\left(t, x_{L}\right)\right) d t= \\
\sum_{i=0}^{P^{t}} \sum_{j=0}^{P^{x}} H_{i j}^{*}\left(\alpha_{n}^{x}\left(x_{R}\right)-\alpha_{n}^{x}\left(x_{L}\right)\right) \int_{T} \alpha_{i}^{t} \alpha_{m}^{t} d t
\end{gathered}
$$

Flux evaluations are highly simplified by using a nodal approach based on Lagrange interpolating polynomials [1], since the flux in a space-time node requires only the degrees of freedom (DOFs) at the nodes occupying the same position in the neighboring elements. To determine the space-time flux, let us define

$$
\left[\left[H_{i j}\right]\right]^{t}=\frac{H_{i j}-H_{i j}^{t,+}}{2} \quad\left[\left[H_{i j}\right]\right]^{x}=\frac{H_{i j}-H_{i j}^{x,+}}{2}
$$

and similarly for the E-field. There, the upper subscript $x,+$ refers to the neighboring node along the spatial boundary (see 


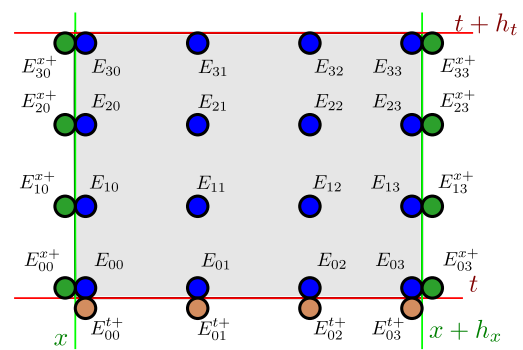

Fig. 1: Notation: a space-time element (in gray) with $P^{t}=P^{x}=3$.

Fig. 1). First, we find the centered version of the numerical fluxes, as the average with the contiguous-space/earlier-time border values

$$
H_{i j}^{\diamond}=\delta_{i 0}\left[\left[H_{0 j}\right]\right]^{t}-\delta_{0 j}\left[\left[H_{i 0}\right]\right]^{x}+\delta_{j P^{x}}\left[\left[H_{i P^{x}}\right]\right]^{x}
$$

and, secondly, we complete them with the lacking terms to obtain upwind fluxes in space, keeping centered fluxes in time

$$
H_{i j}^{\bullet}=H_{i j}^{\diamond}-\delta_{0 j}\left[\left[E_{i 0}\right]\right]^{x} Z^{-1}+\delta_{j P^{x}}\left[\left[E_{i P^{x}}\right]\right]^{x} Z^{-1}
$$

where $\delta_{i j}$ refers to the usual Kronecker-delta, and $Z, Y$ the medium impedance and admittance, respectively (both unity in our case).

A compact matrix formulation can be written by arranging the $E$ and $H$ field coefficients (DOFs) in an ordered columnvector, e.g. $\mathbf{E}=\left[E_{0,0}, \ldots, E_{0, P^{x}} \ldots E_{P^{t}, 0}, \ldots, E_{P^{t}, P^{x}}\right]^{T}$. Eq. (4), with the usual tensor product $\otimes$, becomes

$$
\int_{V} H^{h}(\zeta) \partial_{x} \alpha(\zeta) d \zeta=\mathcal{M}^{t} \otimes \mathcal{C}^{x} \mathbf{H}
$$

with $\mathcal{M}$ and $\mathcal{C}$ being the mass and stiffness matrices, respectively. The spatial-flux term (5) can be expressed as

$$
\begin{array}{r}
\int_{T}\left(H^{h, *}\left(t, x_{R}\right) \alpha\left(t, x_{R}\right)-H^{h, *}\left(t, x_{L}\right) \alpha\left(t, x_{L}\right)\right) d t \\
=\mathcal{M}^{t} \otimes\left(\mathcal{R}_{L}^{x}-\mathcal{R}_{R}^{x}\right) \mathbf{H}^{*}
\end{array}
$$

with $\mathcal{R}_{L}^{t}=\mathbf{e}_{0}^{P^{t}} \mathbf{e}_{0}^{P^{t}, T}$ and $\mathcal{R}_{R}^{t}=\mathbf{e}_{P^{t}}^{P^{t}} \mathbf{e}_{P^{t}}^{P^{t}, T}$ where $\mathbf{e}_{i}^{N}$ is a $N+1$ long zero vector with 1 in entry $i$. Operating similarly for the rest of terms and the Ampere's equation, we can express the scheme locally as

$$
\begin{array}{r}
\varepsilon \mathcal{C}^{t} \otimes \mathcal{M}^{x} \mathbf{E}+\mathcal{M}^{t} \otimes \mathcal{C}^{x} \mathbf{H} \\
=\mathcal{M}^{t} \otimes\left(\mathcal{R}_{L}^{x}-\mathcal{R}_{R}^{x}\right) \mathbf{H}^{*}+\left(\mathcal{R}_{L}^{t}-\mathcal{R}_{R}^{t}\right) \otimes \mathcal{M}^{x} \mathbf{E}^{*}
\end{array}
$$

Let us now assume that our computational domain is divided by $K_{x}$ space-time elements and let us define

$\mathcal{F}_{R, L}^{t}=\mathcal{I}_{K_{x}} \otimes \mathcal{I}_{2} \otimes \mathcal{R}_{R, L}^{t} \otimes \mathcal{M}^{x}, \mathcal{F}_{R, L}^{x}=\mathcal{I}_{K_{x}} \otimes \mathcal{V} \otimes \mathcal{M}^{t} \otimes \mathcal{R}_{R, L}^{x}$

$$
\mathcal{S}^{t}=\mathcal{I}_{K_{x}} \otimes \mathcal{I}_{2} \otimes \mathcal{C}^{t} \otimes \mathcal{M}^{x}, \mathcal{S}^{x}=\mathcal{I}_{K_{x}} \otimes \mathcal{V} \otimes \mathcal{M}^{t} \otimes \mathcal{C}^{x}
$$

with $\mathcal{I}_{N}=\sum_{n=0}^{N-1} \mathbf{e}_{n}^{N-1} \mathbf{e}_{n}^{N-1, T}$ and $\mathcal{V}=\mathbf{e}_{0}^{1} \mathbf{e}_{1}^{1, T}+\mathbf{e}_{1}^{1} \mathbf{e}_{0}^{1, T}$. We can write the following compact expression

$$
\left(\mathcal{S}^{t}+\mathcal{S}^{x}\right) \mathbf{q}=\left(\mathcal{F}_{L}^{t}-\mathcal{F}_{R}^{t}+\mathcal{F}_{L}^{x}-\mathcal{F}_{R}^{x}\right) \mathbf{q}^{*}
$$

where $\mathbf{q}$ represents all the field coefficients in a given spacetime element. The upwind-in-space centered-in-time numerical flux (8) can be expressed as

$$
\mathbf{q}_{\text {upwind }}^{*}=\underbrace{-\mathcal{E}_{R}^{t} \mathbf{q}^{\prime}+\left(\mathcal{E}_{L}^{t}+\mathcal{E}_{L}^{x}-\mathcal{E}_{R}^{x}\right) \mathbf{q}}_{q_{\text {centered }}^{*}}+\left(\mathcal{E}_{L}^{x}-\mathcal{E}_{R}^{x}\right) \tilde{\mathcal{V}} \mathbf{q}
$$

with $\mathbf{q}^{\prime}$ being the state vector of the previous space-time element. The operators $\mathcal{E}$ perform the operations needed to assemble the unknowns associated with the fluxes in the global system of equations. The superscripts $t$ and $x$ and subscripts $L$ and $R$ indicate the boundary at which they are operating. The operator $\tilde{\mathcal{V}}=\mathcal{I}_{K_{x}} \otimes \mathcal{V} \otimes \mathcal{I}_{P^{t}} \otimes \mathcal{I}_{P^{x}}$ indicates the operation on the dual field. Note that $\mathcal{F}^{x} \mathcal{E}^{t} \mathbf{q}^{\prime}=\mathcal{F}^{t} \mathcal{E}^{x} \mathbf{q}=\mathbf{0}$ because $\mathcal{E}$ is defined to extract only the unknowns needed by the flux acting on the boundary indicated by its superscript. Replacing the centered part of (14) into (13)

$\underbrace{\left(\mathcal{S}^{t}+\mathcal{S}^{x}-\mathcal{F}_{L}^{x} \mathcal{E}_{L}^{x}+\mathcal{F}_{R}^{x} \mathcal{E}_{R}^{x}+\mathcal{F}_{L}^{t} \mathcal{E}_{L}^{t}\right)^{-1}\left(-\mathcal{F}_{L}^{t} \mathcal{E}_{R}^{t}\right)}_{\mathcal{H}} \mathbf{q}^{\prime}=\mathbf{q}$

and a similar expression can be formulated for upwind flux. Eq. (15) enables us to find the current state, $\mathbf{q}$, from the previous state, $\mathbf{q}^{\prime}$, in a marching-in-time way. A major limitation of this scheme is its spatial implicitness, requiring matrix inversion (or solving a linear system at each time step) of operators scaling as $\mathcal{O}\left(K_{x}^{2}\right)$ (only tentatively efficient for problems where the space and time scales are very dissimilar).

\section{AN EXPLICIT SCHEME}

Semi-explicit formulations of STDG exist [5], [6], and in this section we present a new explicit alternative simpler than those, in terms of the needed mesh, and using a reduced number of DOFs. A domain-decomposition technique [7] will be used to formulate an explicit variant of the scheme in (15), at the cost of adding a CFL-like causality condition. Rather than solving the whole domain $\Omega$, we divide the problem into smaller regions $\Omega_{d}$ such that $\bigcup_{d}^{N_{d}} \Omega_{d}=\Omega$. Then, we enlarge each region $\Omega_{d}$ to include the elements that are causally connected with it for a given $h_{t}$, we will denote this enlarged region with $\Omega_{d}^{\prime}$. An element is considered to be causally connected with another one if any of its fields at $t$ can propagate to $\Omega_{d}$ at $t+h_{t}$. i. e. if it contains a point within a distance $d \leq c h_{t}$ to any point in $\Omega_{d}$, with $c$ being the numerical speed of light. The border of $\Omega_{d}^{\prime}$ ends in a zero-flux boundary condition that decouples it from the rest of the computational domain. Finally, the problem is solved in each $\Omega_{d}^{\prime}$ using the implicit method described above but passing only the values in $\Omega_{d}$ to the next step. The values in $\Omega_{d}^{\prime} \backslash \Omega_{d}$ are discarded as they are corrupted by the artificial boundary condition. This approach reduces the computational cost from $\mathcal{O}\left(K_{x}^{2}\right)$ to $\sum_{d}^{N_{d}} \mathcal{O}\left(K_{x, d}^{2}\right)$ with $K_{x . d}<K_{x}$. The region sizes $\Omega_{d}$ can be chosen as a trade-off taking into account the computational cost in determining the initial $\mathcal{H}_{d}$, the cost to evolve the scheme, and the size of the time step.

\section{NUMERICAL ANALYSIS}

A PEC-terminated spatial domain has been discretized with $K_{x}=4$ elements of size $h_{x}=0.25$ and order $P^{x}=4$ using the spatial upwind flux (8), as a simple proof-of-concept test-case. The eigenvalues of $\mathcal{H}$ have been numerically found to investigate dispersion and dissipation independently (rather than using error norms such as in [3]-[6]). We have conducted studies for different $h_{t}$, which have not exhibited eigenvalues 
with positive real parts, thus implying that the scheme is unconditionally stable for any $h_{t}$.

Fig. 2 shows the dispersion and dissipation properties of the scheme for the first resonant modes to the closest analytical mode $k_{\mathrm{a}}=\pi$ with $\omega_{\mathrm{a}}=\pi$ for different STDG schemes and a classical DG evolved using LSERK4 and the same upwind spatial fluxes. The accuracy of the scheme presents high-order convergence with $h_{t}$, following the relationship $\left(h_{t}^{2 P_{t}+1}\right)$ for the dissipation, and $\left(h_{t}^{2 P_{t}+2}\right)$ for the dispersion, in agreement with [8] for the spatial DG semi-discrete scheme. Therefore we can conclude that the spatial and temporal convergences of the scheme coincide. Note that, although the LSERK4 physical eigenvalue can be computed and represented, it is unstable for approx. $h_{t} \geq 0.05$ because of the presence of other eigenvalues lying outside its stability region, thus limiting with $h_{t, \max } \propto$ $\left(P^{x}\right)^{-2} \min h_{x}^{k}$. We also find that we have higher convergence for $P^{t} \geq 2$, which is a significant improvement over LSERK4, especially when combined with a higher $P^{x}$.
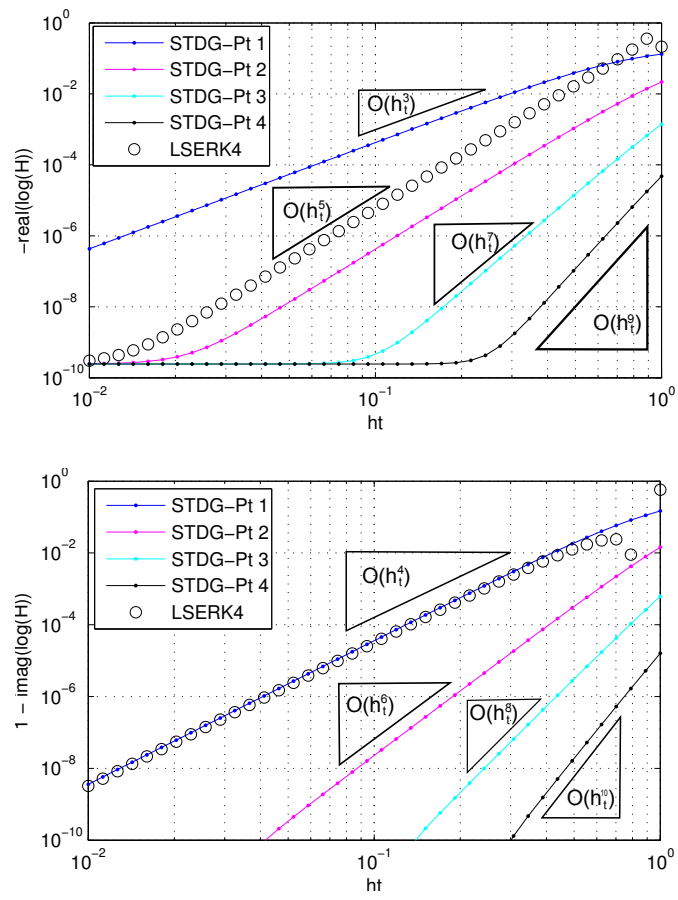

Fig. 2: Dissipative (up)/dispersive (down) convergence rates of the eigenvalues of the evolution operator $\mathcal{H}$ for different orders $\& h_{t}$.

\section{Numerical Results}

For validation, we have simulated the above problem using a discretization of $K_{x}=8, h_{x}=0.125, h_{t}=0.1$, and $P^{t}=P^{x}=4$ and upwind fluxes up to a time $T=10000$ (note that all quantities are dimensionless in our system of units). Also, we have computed the same problem with the explicit implementation of the scheme. The STDG scheme has a single evolution operator with 6400 non-zero entries. The E-STDG works with a split domain having 8 different evolution operators totaling 4800 non-zero entries, indicating a significant reduction in the computational complexity. Fig. 3 shows the resonances and the error for the first modes.

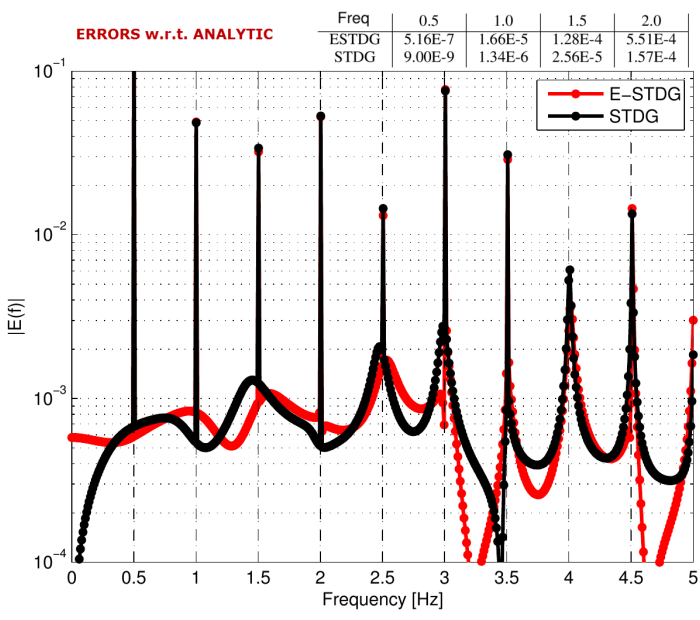

Fig. 3: Response to a white noise in the E-STDG and STDG schemes with $P^{t}=4, h_{t}=0.1, P^{x}=4, K_{x}=8, h_{x}=0.125$ after a time of 100 . Vertical dashed lines represent the analytical modes.

\section{Conclusions}

In this work, we have introduced and analyzed a novel implicit and stable nodal STDG technique, and compared it with the classic DG-LSERK4 scheme. This nodal formulation needs to store twice the DOFs of the LSERK4 formulations, but it is suitable to be used in high-order-in-space schemes, taking full advantage of the convergence of the spatial DG semi-discretization. To overcome its implicitness, we have proposed a new explicit implementation, showing that the scheme can be computationally affordable, allowing certain freedom in choosing the size of the time step and evolution operators.

\section{REFERENCES}

[1] J. S. Hesthaven and T. Warburton, Nodal Discontinuous Galerkin Methods: Algorithms, Analysis, and Applications, 1st ed. Springer Publishing Company, Incorporated, 2007.

[2] M. Liu, K. Sirenko, and H. Bagci, "An efficient discontinuous Galerkin finite element method for highly accurate solution of Maxwell equations," Antennas and Propagation, IEEE Transactions on, vol. 60, no. 8, pp. 3992-3998, 2012.

[3] S. Petersen, C. Farhat, and R. Tezaur, "A spacetime discontinuous Galerkin method for the solution of the wave equation in the time domain," International Journal for Numerical Methods in Engineering, vol. 78, no. 3, pp. 275-295, 2009. [Online]. Available: http://dx.doi.org/10.1002/nme.2485

[4] J. van der Vegt and $H$. van der Ven, "Space-time discontinuous Galerkin finite element method with dynamic grid motion for inviscid compressible flows: I. general formulation," Journal of Computational Physics, vol. 182, no. 2, pp. 546 - 585, 2002. [Online]. Available: http://www.sciencedirect.com/science/article/pii/S0021999102971858

[5] P. Monk and G. R. Richter, "A discontinuous Galerkin method for linear symmetric hyperbolic systems in inhomogeneous media," Journal of Scientific Computing, vol. 22-23, pp. 443-477, 2005. [Online]. Available: http://dx.doi.org/10.1007/s10915-004-4132-5

[6] R. S. Falk and G. R. Richter, "Explicit finite element methods for symmetric hyperbolic equations," SIAM J. Numer. Anal., vol. 36, no. 3, pp. 935-952, Mar. 1999. [Online]. Available: http://dx.doi.org/10.1137/ S0036142997329463

[7] J. C. Meza and S. W. W., "Domain decomposition algorithms for linear hyperbolic equations," Rice University, Tech. Rep., 1987.

[8] J. Alvarez, L. D. Angulo, M. R. Cabello, A. R. Bretones, and S. S. G. Garcia, "An analysis of the leap-frog discontinuous galerkin method for maxwell's equations," Microwave Theory and Techniques, IEEE Transactions on, vol. 62, no. 2, pp. 197-207, Feb 2014. [Online]. Available: http://dx.doi.org/10.1109/TMTT.2013.2295775 